\title{
Expression of the NS3 protein of hepatitis C virus used in the HCV UMELISA® test kit
}

\begin{abstract}
To manufacturing the HCV UMELISA ${ }^{\circledR}$ Test kit, a recombinant NS3 protein is used as part of the antigen mixture. This protein is obtained from Escherichia coli XL-1Blue transformed with pR2M6-NS3-His plasmid downstream trp promoter. Regulation of expression has been identified as the main cause that affects yield and reproducibility at the fermentation process. To improve protein expression and transcription regulation, cloning of NS3 sequence was performed downstream tac promoter. At the same time, three $E$. coli strains (XL 1-Blue, W3110 and C600) were used to assess the expression of the NS3 protein. The tac promoter improved the expression level at all the strains evaluated from $20 \%$ under trp until $30 \%$ and a reduction of promoter leakage before induction was observed compared with trp promoter. Due to the insensibility to the high metabolic burden, C600 was settled as the strain to use for NS3 expression. The new construction was well recognized by a pool of positive sera and individual sera from infected humans in an indirect ELISA using the HCV UMELISA ${ }^{\circledR}$ Test kit.
\end{abstract}

Keywords: HCV, C virus, ELISA, UMELISA, E. coli, hepatocellular, carcinoma
Volume 8 Issue 2 - 2020

\author{
José Miguel Fernández Torres,' Yeosvany \\ Cabrera Artiles,' María Teresa Barcelló \\ Ávila,' Ronny Camacho Pupo, ${ }^{2}$ Duniesky \\ Martínez García,' Alina Sobrino León' \\ 'Department of R \& D, Center for Genetic Engineering and \\ Biotechnology, Cuba \\ 2Department of Quality Control, Center for Genetic \\ Engineering and Biotechnology, Cuba
}

\begin{abstract}
Correspondence: José Miguel Fernández Torres, Department of R \& D, Center for Genetic Engineering and Biotechnology, Cuba, Tel +534l326273, Fax 4I328539,
\end{abstract} Email jose.fernandez@cigb.edu.cu

Received: June 10, 2020 | Published: July 20, 2020

\section{Introduction}

Hepatitis $\mathrm{C}$ is a disease that affects people causing liver cirrhosis and hepatocellular carcinoma. Its etiologic agent is an RNA virus named Hepatitis $\mathrm{C}$ virus (HCV). ${ }^{1}$ Screening for HCV infection is based on the detection of anti-HCV antibodies, and confirmed by the presence of HCV RNA to identify patients with ongoing infection. ${ }^{2}$ These tests are crucial to analyze the magnitude of the pandemic in different regions and design public health interventions. The NS3 protein of $\mathrm{HCV}$ is a multifunctional enzyme with serine-protease activity at the N-terminal end, and helicase activity at the rest of their structure that prevents coiling of RNA duplex strands formed during viral replication. ${ }^{3}$ In assays based on antibodies detection, the NS3 protein had been included due to its improvements in sensitivity and specificity on second generation tests. ${ }^{4}$ In Cuba, the ELISA for the screening of patients (HCV UMELISA ${ }^{\circledR}$ Test kit) is developed by the Center of Immunoassays. In this kit, an important biological reagent is a recombinant fragment of NS3 produced by the Center for Genetic Engineering and Biotechnology of Sancti-Spiritus. This protein is obtained from Escherichia coli XL-1Blue transformed with pR2M6-NS3-His plasmid downstream trp promoter on Luria-Bertani broth (LB). Regulation of expression has been identified as the main cause that affects yield and reproducibility at the fermentation process which leads to affect purification process. There are a few promoters that could be used to avoid leakage and to increase protein expression in complex media, like tac promoter. ${ }^{5}$ In this manuscript, we report the expression of this NS3 fragment downstream tac promoter to improve expression and promoter regulation in complex media.

\section{Materials and methods}

\section{Escherichia coli strains}

XL1-blue: endA1 gyrA96(nalR) thi-1 recA1 relA1 lac glnV44 F'[:: Tn10 proAB+ lacIq (lacZ)M15] hsdR17(rK- mK+).

W3110: F- lambda- IN(rrnD-rrnE)1 rph-1

C600: F- tonA21 thi-1 thr-1 leuB6 lacY1 glnV44 rfbC1 fhuA1

\section{Cloning the NS3-tac gene}

The gene, encoding a stabilizing IL-2 fragment at the 5' end linked to a fragment of HCV NS3 sequence, was extracted from pR2M6-NS3-His plasmid using the 5' (aattccatggcgcctacttcaagttc) and $3^{\prime}$ (taagtcgacgcaggtgttgcaatcaat) oligonucleotides by PCR. The DNA fragment obtained was sub-cloned into the commercial vector pGEM-Teasy. Cloning of NS3- tac gene was released in Nco I and Sal I sites of PTBAD vector, which contains a polyhistidine tail at the Sal I site, digested previously with the respective enzymes (Nco I and Sal I) and dephosphorylated. The ligation reaction was done using T4 DNA ligase from pGEM-Teasy kit. So, the final construction would have incorporated a stabilizing IL-2 fragment of 60 amino acids in the protein and a polyhistidine tail for purification purposes. All cloning steps were carried out in Escherichia coli X1-1 Blue. Grow kinetics of E. coli in $50 \mathrm{~mL}$ shaking cultures and expression of recombinant protein Il2-NS3-His. The pR2M6-NS3-His and PTBADIL2-NS3-His vectors were used for the expression of IL2-NS3-His to evaluate expression of the protein under both trp and tac promoters respectively in three strains (XL-1Blue, W3110 and C600) on LB medium. PTBAD plasmid was used as negative control of expression. Inoculation in $5 \mathrm{ml} \mathrm{LB}$ medium supplemented with ampicillin $(100 \mu \mathrm{g} / \mathrm{mL})$ was carried out with a single colony of the transformed strain with the corresponding plasmid and leaving it to grow at $37^{\circ} \mathrm{C}$ overnight. Then, $0.5 \mathrm{ml}$ of the culture was inoculated into $50 \mathrm{~mL}$ of the $\mathrm{LB}$ medium supplemented with ampicillin and incubated at $37^{\circ} \mathrm{C}$ for $12 \mathrm{~h}$ while shaking at $250 \mathrm{rpm}$. Induction of expression was done when cultures rises an optical density at $620 \mathrm{~nm}\left(\mathrm{OD}_{620 \mathrm{~nm}}\right)$ of $0.4-0.6$, using IPTG $(0.5 \mathrm{mM})$ for PTBAD and PTBAD-IL2-NS3-His plasmids and $\beta$-Indoleacrilic acid $(0.21 \mathrm{mM})$ for pR2M6-NS3-His vector. Samples were collected periodically for evaluation.

\section{SDS-page}

Protein electrophoresis was performed on a $12 \%$ polyacrylamide denaturing gel with $2 \%$ Sodium Dodecyl Sulfate (SDS). Samples were diluted in loading buffer $(125 \mathrm{mM}$ Tris- $\mathrm{HCl}, \mathrm{pH} 6.8,1 \%$ (w/v) SDS, $5 \%(\mathrm{v} / \mathrm{v})$ glycerol, $10 \mathrm{mM}$ Dithiothreitol, $0.005 \%(\mathrm{w} / \mathrm{v})$ Blue 
Bromophenol) and heated for $10 \mathrm{~min}$ at $100{ }^{\circ} \mathrm{C}$. The samples were run for about $45 \mathrm{~min}$ at a constant voltage of $200 \mathrm{~V}$ in running buffer (25mM Tris-HCl, $\mathrm{pH} 8.8 ; 192 \mathrm{mM}$ Glycine; $35 \mathrm{mM}$ SDS). The gel was stained with a $0.1 \%(\mathrm{w} / \mathrm{v})$ Coomassie R-250 solution, $40 \%$ $(\mathrm{v} / \mathrm{v})$ methanol, $10 \%(\mathrm{v} / \mathrm{v})$ acetic acid for $20 \mathrm{~min}$ and the bands were visualized after gel destaining with $10 \%(\mathrm{v} / \mathrm{v})$ methanol and $10 \%(\mathrm{v} / \mathrm{v})$ acetic acid.

\section{Detection of proteins by western blot}

In Western blot assay, the proteins from the samples were transferred from the polyacrylamide gel to an extra Hybond-C nitrocellulose membrane (Amersham Life Science, UK) for immunodetection. As primary antibody, three monoclonal antibodies were used and dissolved at a concentration of $10 \mu \mathrm{g} / \mathrm{mL}$ in a solution of skim milk $(1 \% \mathrm{w} / \mathrm{v})$ - PBS-Tween $20(0.05 \% \mathrm{w} / \mathrm{v})$. As the secondary antibody, an alkaline phosphatase conjugated mouse anti-IgG antibody (Sigma Aldrich, USA) diluted 1: 20000 (Sigma Aldrich, USA) was used. Colorimetric detection was performed with 5-bromo-4-dichloroindolyl phosphate / nitroblue tetrazolium (BCIP/NBT).

\section{IMAC purification}

From the total biomass obtained in a $500 \mathrm{ml}$ LB medium shaking flask of a growth culture, $0.6 \mathrm{~g}$ were ruptured by sonication and centrifuged (10000rpm). Then the pellet was collected and solubilized $(10 \mathrm{ml} / \mathrm{g}$ biomass) for $1 \mathrm{~h}$ in binding buffer $\mathrm{pH} 8(8 \mathrm{M}$ urea, $100 \mathrm{mM}$ sodium phosphate) and filtered by $0.45 \mu \mathrm{m}$. Once the matrix was equilibrated, the sample was passed through a "His-Select®" (GE Health care) matrix packaged in a C 10/10 column (GE Health care) with a height of $5 \mathrm{~cm}$. Elution was performed with the same buffer but at $\mathrm{pH}$ 6. The volumetric flow was kept constant at $0.6 \mathrm{ml} / \mathrm{min}$ throughout the process. The chromatographic profile was monitored by measuring absorbance at $280 \mathrm{~nm}$. Denaturing electrophoresis in $12 \%$ polyacrylamide gel was used as a criterion of purity using image J V.0.5 program and protein concentrations was determined by BCA using bovine albumin as standard. ${ }^{6}$

\section{ELISA for sensibility and specificity of recombinant NS3}

To determine the sensitivity and specificity in the HCV UMELISA ${ }^{\circledR}$ Test kit we substituted the NS3 used in this kit by the purified NS3 expressed under tac promoter, in the mixture of antigens used in the coating solution. First was determined the optimum working concentration of the protein of interest that was of $1 \mu \mathrm{g} / \mathrm{mL}$ and later were coated the plates using the mixture of antigens, and following all the recommended specifications to produce the diagnostic kit. For specificity, we used samples of negative sera from blood donors of the Havana Provincial Blood Bank. All samples were considered positive when the ratio (Fm-B)/ (P-B) was higher than or equal to 0.27 . Where Fm, $\mathrm{P}$ and $\mathrm{B}$ refer to the fluorescence of the sample evaluated, the positive control concerning the HCV UMELISA ${ }^{\circledR}$ Test kit, and the blank, respectively. The sensitivity of antibody detection was estimated as the percentage of samples considered to be positive with the new NS3-tac protein concerning those considered to be positive with the NS3-trp referenced by a commercial kit (ORTHO ${ }^{\circledR}$ HCV 3.0). For this, 16 samples from seroconversion panels PHV 919 and 920 from SeraCare ${ }^{\circledR}$ were used. Specificity was defined as the percentage of negative HCV samples that were considered negative with the NS3-tac protein, compared to those considered negative with the NS3-trp protein in the HCV UMELISA ${ }^{\circledR}$ Test kit.

\section{Results and discussion}

PCR products were analyzed on an agarose gel in parallel with Lambda Hind III-EcoRI DNA size marker and the expected 995bp fragment was observed (Figure 1A). Sequencing analysis was done to confirm amplification. At the PCR reaction, 12 amino acids were removed from the NS3 protein codified by pR2M6-NS3-His vector because it does not belong to viral NS3 sequence. The ligation reaction was done for cloning the amplified region into PTBAD vector. NS3 protein was expressed under both, trp promoter and tac promoter. A western-blot was performed for protein identification (Figure 1C). All fragments of the quimeric NS3 protein were recognized by the antibodies used on the assay. This showed that cloning NS3 under tac promoter was successful (Figure 1). A significant alteration on SDS-PAGE mobility between both proteins was observed. The NS3 expressed under tac promoter regulation migrated less than NS3 protein expressed downstream trp promoter although we expected the contrary. This anomalous migration pattern could be caused by altered SDS loading on each protein due to differences on the helicity at the C-terminal region among both proteins. ${ }^{7}$

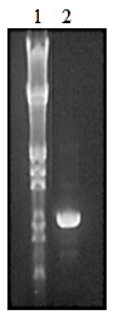

$\mathbf{A}$

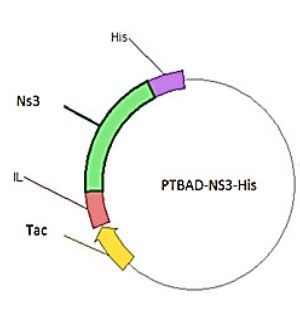

B

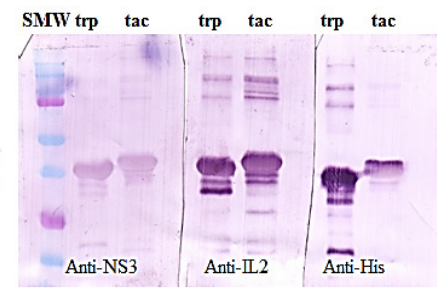

C
Figure I (A): PCR amplification of NS3 sequence. Line I: lambda Hind III marker, line 2: PCR product (B): Scheme of the final vector for NS3 expression downstream tac promoter. (C):Western blot performed with NS3 transferred to a PVDF membrane and recognized by three monoclonal antibodies (The antibodies used are referred in the figure). SMW: Standard molecular weight. trp: quimeric NS3 protein expressed downstream trp promoter. tac: quimeric NS3 protein expressed downstream tac promoter.

\section{NS3 expression}

For evaluation of protein expression under both promoters, three strains were used as hosts and grown in $50 \mathrm{ml}$ shaking flasks (Figure 2 ). A reduction at growth rate was observed in the three strains (XL-1Blue, W3110 and C600) after promoter induction. The higher effect of protein expression on the final cell yield was observed on $\mathrm{XL}-1 \mathrm{Blue}$ strain. This is due to XL-1Blue has thi-1 mutation which impedes it to grow well without B1 vitamin. The same effect was seen on either W3110 and C600 strains but less than for XL. This growth decrease has been well described and is associated with the high metabolic burden when protein expression is achieved under strong promoters. ${ }^{8}$ The NS3 expressed under trp promoter in XL strain was $20 \%$ from total proteins like ${ }^{4}$ described. Protein expression under tac promoter was higher than $30 \%$ at each strain, whereas that downstream trp promoter was observed only an average of $20 \%$ in each strain. Other researchers have described too bigger expression with $t a c$ promoter than for trp promoter. ${ }^{5}$ Besides, the tac promoter allowed less protein production before induction than trp promoter in all strains assessed, showing better regulation on NS3 expression. The best regulation was observed in C600 strain when expressed NS3 downstream tac promoter with only $4.7 \%$ of protein leaking before 
induction while the worse regulation under tac promoter was observed on XL strain with $17.5 \%$. This leakage on XL was not expected due to a lac Iq mutation present in their genotype. However, it is known that several amounts of lactose may be present in yeast extract and the tryptone. ${ }^{9}$ The lesser leaking of NS3 expression before induction in C600 strain is associated with a lacY mutation in their genotype that prevent lactose permease synthesis and hence impede leakage corresponding to lactose induction. In terms of productivity, C600 showed major rate in NS3 production continued by W3110. The high expression, insensibility to big metabolic burden, great regulation of expression and major grow rate, settled $\mathrm{C} 600$ as the best strain for production of NS3 protein in LB medium downstream tac promoter.

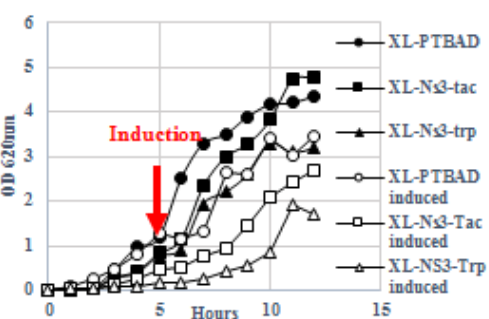

A

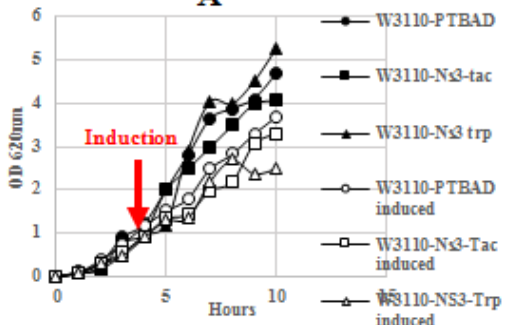

B

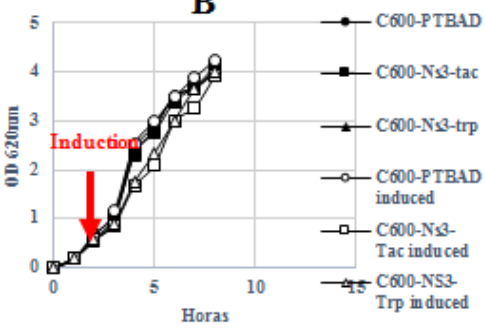

C

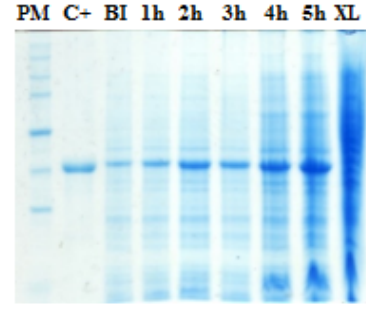

D

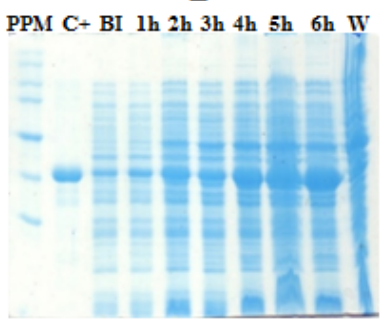

E

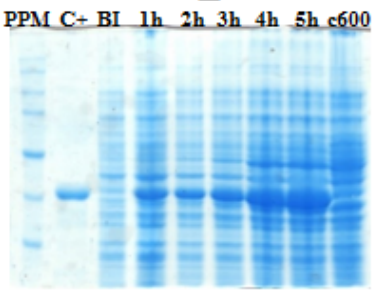

F

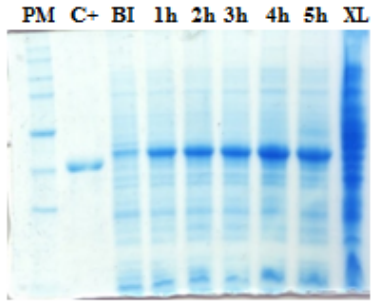

G

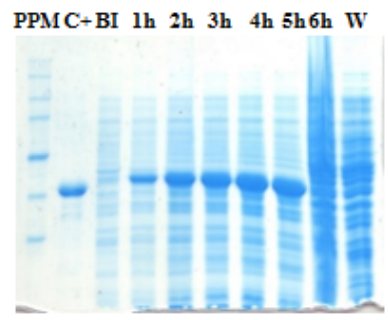

H

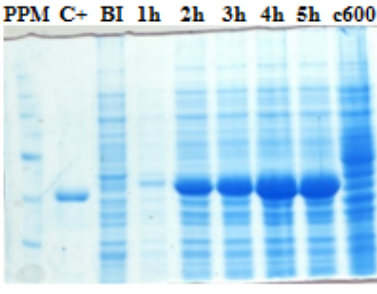

I

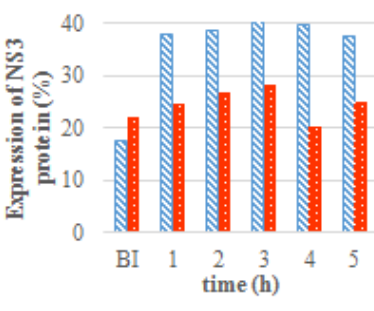

J

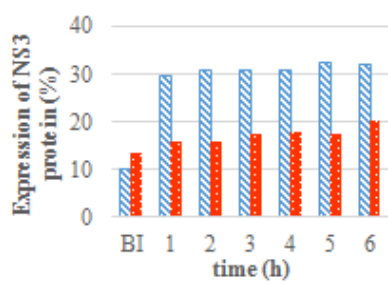

K

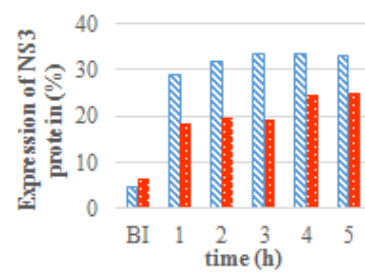

L

Figure 2 A \& B \& C: Growth kinetics in shaking flasks of the E. coli strains XL-IBLUE,W3IIO and C 600 respectively. D \& E \& F: Polyacrylamide gel of the NS3 expressed downstream trp promoter in XL,W3IIO and C600 strains respectively. G \& H \& I: Polyacrylamide gel of the NS3 expressed downstream tac promoter in XL,W3 II 0 and C600 strains respectively (SMW: Broad range Protein marker, Promega, USA. C+: Purified NS3 used as a reference. BI: Sample was taken before induction. Ih, $2 \mathrm{~h} . . .5 \mathrm{~h}$ : Hours after induction. XL: Xl- IBlue strain transformed with the plasmid without the insert. W:W3III0 strain transformed with the plasmid without insert. C600: C600 strain transformed with the plasmid without the insert).J \& K \& L: Comparison of NS3 expression downstream both promoter (trp promoter in red and tac promoter in blue) in XL,W3II0 and C600 respectively.

\section{IMAC purification of NS3 protein}

In diagnostic assays, the purity of antigens is crucial for avoiding cross-linking of antibodies from the sera. The new NS3 (NS3 Tac) was purified using metal affinity chromatography through the 6xHis tag attached to the $\mathrm{C}$-terminus protein. Previously described in Palenzuela et $\mathrm{al}^{4} \mathrm{NS} 3$ protein was expressed as a cytoplasmic inclusion body. Due to the insoluble nature of the expressed NS3 protein, urea was used as a denaturant. The protein eluted to $\mathrm{pH} 6$ with a recovery of $59.9 \%$. The purity obtained from the NS3-tac (97\%) is advantageous in the diagnostic test where the purity of antigens is required (Figure 3 ).

\section{Evaluation of the sensitivity and specificity of the recombinant protein}

To assess the functionality of the protein, an HCV UMELISA ${ }^{\circledR}$ Test kit was used with the NS3 expressed under either tac or trp promoters coated both in a 96 well plate. For its recognition, the positive and negative sera supplied with the kit were used. As control was used a coated plate present in the kit, where the coating is performed with NS3 but also with other proteins of the nucleocapsid. Although a few amino acids were removed from the original sequence, was not observed differences between both proteins (Figure 4). Besides, a set of HCV positive samples and negative HCV samples from seroconversion panels PHV 919 and PHV 920 HCV SeraCare were used for its assessment. Here was used the purified NS3 protein (NS3 Tac) as a part of the antigen mixture in the coating solution and a reference mixture containing the NS3 obtained by the old strategy (NS3 downstream trp promoter). As control of specificity criteria, a commercial ORTHO HCV 3.0 kit was used. The sensitivity of UMELISA with the new NS3 protein was $100 \%$ in 16 samples of the PHV 919 and $920 \mathrm{HCV}$ panels referenced by the ORTHO $\mathrm{HCV}$ 
3.0 kit (Table 1). These results show that the subtraction of the noun related amino acids on the NS3 protein does not affect the sensitivity and specificity in the HCV-UMELISA Test kit, therefore the new NS3 protein can be used in HCV diagnostic (Table 1).

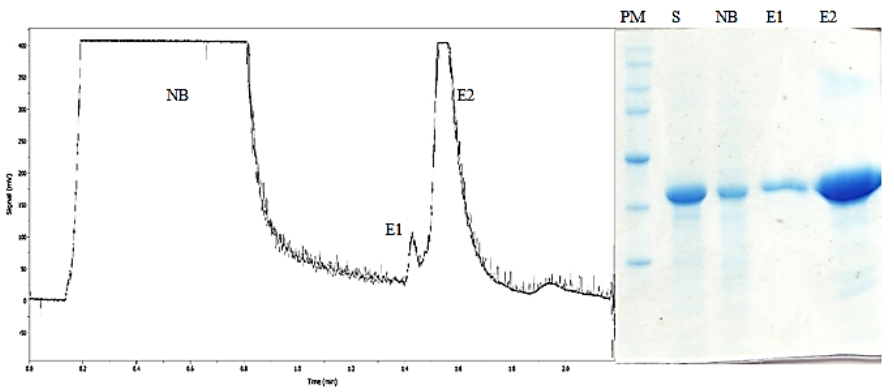

Figure 3 Chromatogram (A) and polyacrylamide gel of an IMAC purification of NS3 protein expressed downstream tac promoter in C600 strain. NB: Noun bound fraction. EI and E2: Fractions eluted at $\mathrm{pH} 6$. PM: Broad range protein marker, Promega, USA. S: Sample before loading into the column.

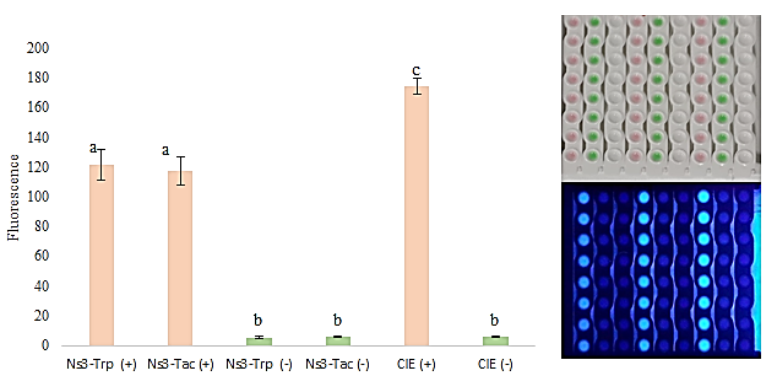

Figure 4 UMELISA performed with both NS3 expressed downstream both trp (NS3-Trp) and tac (NS3-Tac) promoters using the UMELISA HCV kit developed by Center of Immunoassays, Cuba. The negative and positive controls supplied with the kit were used and fluorescence values were compared by a test $\mathrm{t}(\alpha<0,05)$

Table I Comparison of serum diagnostic results obtained with the NS3 Trp and NS3 Tac proteins, showing the coating of HCV UMELISA Test kit and the certified sero conversion panels PHV 919 and 920 from Sera Care ${ }^{\circledR}$

\begin{tabular}{llllll}
\hline Panel code & NS3trp (Reference) & & NS3 tac(New) & ORTHO HC:V 3.0 \\
\hline PHV 919-1 & $0 \_07$ & Neg & 0.06 & Neg & Neg \\
PHV 919-2 & 0.09 & Neg & 0.07 & Neg & Neg \\
PHV 919-3 & 0.07 & Neg & 0.06 & Neg & Neg \\
PHV 919-4 & $0 \_10$ & Neg & 0.08 & Neg & Neg \\
PHV 919-5 & 1.04 & Pos & 0.94 & Pos & Pos \\
PIIV 919-6 & 138 & Pos & 1.26 & Pos & Pos \\
PHV 919-7 & 126 & Pos & 1.19 & Pos & Pos \\
PHV 920-1 & $0 \_05$ & Neg & 0.04 & Neg & Neg \\
PHV 920-2 & 0.11 & Neg & 0.09 & Neg & Neg \\
PHV 920-3 & $0 \_52$ & Pos & 0.59 & Pos & Neg \\
PHV 920-4 & 1.04 & Pos & 0.92 & Pos & Neg \\
PHV 920-5 & 97 & Pos & 0.93 & Pos & Pas \\
PHV 920-6 & 12 & Pos & 0.94 & Pos & Pos \\
PHV 920-7 & 13 & Pos & 139 & Pos & Pos \\
PHV 920-1 & 134 & Pos & 1.13 & Pos & Pos \\
PHV 920-9 & 32 & Pos & 33 & Pos & Pos \\
\hline
\end{tabular}

Relative fluorescence values were calculated as the (Fm-B)/(P-B) ratio(the samples are considered positives when this ratio is superior than 0.3$)$, where $\mathrm{Fm}, \mathrm{P}$ and $B$ stand for the fluorescence of the samples, the positive control of the HCV UMELISA assay, and the blank, respectively. The presented values stand for the mean values of two replicates per serum tested

\section{Conclusion}

A new genetic construction was established, which enabled more than $30 \%$ expression of the NS3 protein at the insoluble form in $E$. coli through genetic regulation via the tac promoter which avoided leaking of expression before induction comparing to $\operatorname{trp}$ promoter
Between the three strains assessed here, $\mathrm{C} 600$ was settled as the best host for NS3 expression downstream tac promoter. The new NS3 was successfully purified by IMAC and showed good activity in immunoassays when it was compared with the old NS3 used in HCV diagnostic by the HCV UMELISA ${ }^{\circledR}$ test kit. ${ }^{10}$ 


\section{Acknowledgements}

None.

\section{Conflicts of interest}

The author declares that there is no conflict of interest.

\section{References}

1. Puchades LR, Berenguer M. Introduction to hepatitis $C$ virus infection: Overview and history of hepatitis $\mathrm{C}$ virus therapies. Hemodial Int. 2018;22:S8-S21.

2. European Association for the Study of the Liver. EASL recommendations on treatment of hepatitis C 2018. J Hepatol. 2018;69:461-511.

3. Raney KD, Sharma SD, Moustafa IM, et al. Hepatitis C Virus Nonstructural Protein 3(HCVNS3): A Multifunctional Antiviral Target. Int $J$ Biol Chem. 2010;285(30):22725-22731.

4. Palenzuela DO, Pedroso S, Roca J, et al. A new NS3 recombinant protein shows improved antigenic properties for HCV diagnosis. Biotecnol Apl. 2006;23(2):94-98.
5. De Boer H, Comstock LJ, Vasser M. The tac promoter: A functional hybrid derived from the trp and lac promoters. Proc Natl Acad Sci USA.1983;80:21-25.

6. Smith PK. Measurement of protein using bicinchoninic acid. Anal Biochem. 1985;150(1):76-85

7. Rath A, Glibowick M, Nadeau VG, et al. Detergent binding explains anomalous SDS-PAGE migration of membrane proteins. PNAS. 2009; 106 (6): 1760-1765.

8. Perry ChC. Engineering cell physiology to enhance recombinant protein production in Escherichia coli. Appl Microbiol Biotechnol. 2007;76:521532.

9. Studier FW. Protein Production by auto-induction in high-density shaking cultures. Protein expression and purification. 2005;(41):207-237.

10. Rosano GL, Ceccarelli EA. Recombinant protein expression in Escherichia coli: advances and challenges. Frontiersin. 2014. 